\title{
Online Survey: Prevalence and Attitude of Jordanians Towards Using Herbal Remedies in the Pandemic COVID-19
}

\author{
Noha Abd Alkareem Younis Younis', Rania Mohammad Hamam²,*, Amal Mayyas ${ }^{3, *}$
}

\begin{abstract}
Noha Abd Alkareem Younis Younis', Rania Mohammad Hamam $^{2, *}$, Amal Mayyas ${ }^{3, *}$
\end{abstract}

'Department of Pharmacy, Al Balqa Applied University, Aqaba Universal College, Aqaba, JORDAN.

${ }^{2}$ Department of Pharmacy, Princess Sarvath Community College, Amman, JORDAN. ${ }^{3}$ Department of Pharmacy, Faculty of Health Sciences, American University of Madaba, Madaba, JORDAN.

\section{Correspondence}

\section{Rania Mohammad Hamam}

Department of Pharmacy, Princess Sarvath Community College, Amman, JORDAN.

Phone Number: 00962798321806

E-mail: r.hamam@pscc.edu.jo

\section{Amal Mayyas}

Department of Pharmacy, Faculty of Health Sciences, American University of Madaba, Madaba, JORDAN.

Phone Number: 00962795561800

E-mail: a.mayyas@aum.edu.jo

History

- Submission Date: 25-09-2021;

- Review completed: 26-10-2021;

- Accepted Date: 02-11-2021.

DOI : 10.5530/pj.2021.13.210

Article Available online

http://www.phcogj.com/v13/i6

\section{Copyright}

(c) 2021 Phcogj.Com. This is an openaccess article distributed under the terms of the Creative Commons Attribution 4.0 International license.

\section{ABSTRACT}

Coronavirus disease 2019 (COVID-19) is a new virus caused by SARS-CoV-2, a coronavirus from the coronavirus family, that can provoke severe respiratory syndrome. The disease became a pandemic in December 2019. Impaired immune modulation is one of the processes implicated in its pathogenesis, which contributes to poor COVID-19 outcomes. Many investigations have been conducted using medication to control coronaviral infections as antiviral agents or immunomodulators. The results of these tests, however, demonstrated that these medications were useless in fighting the disease. Some people believe that using herbal immunomodulators can aid in the prevention or even curing of COVID-19. Based on prior research, in the management of COVID-19, several botanicals may be useful. We go over the patient attitude and benefits of using such herbal remedies in the regimen of treating COVID-19 in Jordan. Key words: Coronavirus, COVID-19, SARS-CoV-2, Herbal immunomodulators, Pandemic, Herbal remedies, Botanicals.

\section{INTRODUCTION}

Coronaviruses are single-stranded RNA viruses that can cause respiratory, gastrointestinal, neurological, and hepatic diseases in both animals and humans. ${ }^{1}$

On December 30, 2019, the novel coronavirus made its first appearance in the Wuhan, China. ${ }^{2}$

The World Health Organization renamed this causative virus coronavirus 2 and the related disease COVID-19 for the time being and declared it as a pandemic disease. ${ }^{3}$

The disease continues to spread quickly mostly through intimate contact with infected people via respiratory droplets from sneezing or coughing. additionally, there are two alternative routes to spread the virus: touch and aerosol transmission. ${ }^{4}$

COVID-19 causes a range of unspecific symptoms in infected patients, the foremost common symptom reported by patients is fever, followed by cough, sputum production, fatigue, loss of taste, and smell sense and headache. ${ }^{5}$

In addition, certain fatal cases are described in individuals who are stricken by increasing respiratory failure as a results of virus activity attacking alveolar epithelial cells. Moreover, according to a new study, the virus causes the creation of antibodies that circulate in the blood, producing clots in COVID-19 patients. $^{6}$

WHO created master and generic protocols to guide members in the development of clinical studies to evaluate claims of effective COVID-19 treatment.

Herbal medications are not the same as traditional medicine. They are not required to meet premarketing requirements. For traditional medicine, there are safety and effectiveness requirements that must be met. ${ }^{7,8}$
Herbal medicine use has risen dramatically across the world, with many people now using these items for the treatment of a variety of health conditions in healthcare settings in various countries. ${ }^{9,10}$

Herbal medication is reported to be used for a variety of purposes, including disease prevention, and treatment of chronic diseases such as dyslipidemia, hypertension, diabetes and cancer. ${ }^{11,12}$

In Arab communities, herbal medicines are used by around $80 \%$ of the population for disease prevention and care. ${ }^{13}$

Herbal medicines in Jordan have remained common due to historical, cultural, and psychosocial factors. Herbal medicine is widely used for a wide range of ailments such as it is less expensive, more closely aligns with the patient's values, reduces concerns about chemical (synthetic) drug side effects, meets a need for more individualized health care, and allows for a more personalized approval process. ${ }^{14}$

The goal of this study was to investigate the prevalence and attitudes toward herbs and natural products usage among Jordanians during the coronavirus pandemic in 2021, as well as to discover factors that influence herbal remedies use.

Herbs will aid in the preparation of interventions to raise herbal usage awareness. This may also highlight the importance of establishing mechanisms to govern herbal medicine entry, delivery, and usage. ${ }^{15}$

The questionnaire was sent in Arabic to be suitable to all classes of society.

The questionnaire was divided into three sections: the first part was about data of social demographics such as age, gender, educational level, financial capacity, and finally the health condition and previous medical history.

The second part was about the covid 19 infection. The third part is about herbal types and the use of plants consumed by participants. 
The last part is about participants beliefs and knowledge about herbal medicinal plants.

At the time of the study, the Jordanian Ministry of Health has recorded that the total number of cases in Jordan raised to $(733,198)$ daily and the total number of deaths to (9407). The total number of recoveries in home isolation and hospitals verified of COVID-19 was 712,929.

Until now, no definite treatment for COVID-19 management has been recommended for people. COVID-19 is currently being treated and prevented by developing vaccines and particular therapeutic drugs that target SARS-CoV-2. ${ }^{16}$

As a therapy for COVID-19, some of the prospective therapeutic agents were used in most contourites of the world like antiviral medicines such as Remdesivir, chloroquine/hydroxychloroquine, and dexamethasone. ${ }^{17}$

The Jordan health authorities approved the use of antimalarial medicines to treat new coronaviruses in Jordanian hospitals. The treatment protocol was giving "Hydroxychloroquine," "Azithromycin," "Paracetamol," "Zinc sulfate," and "Vitamin C" in order to handle the active disease in military and governmental hospitals in Jordan.

The study's goals were to assess Jordanians' beliefs and knowledge regarding herbal medicinal use during covid 19 infection.

\section{METHODOLOGY}

The current study is a cross-sectional online study conducted in Jordan. The data was collected from September 1 to January 4 of 2021. Google Forms was used to collect data online. During the research period, 540 Jordanians were participated and provided feedback in this study.

A questionnaire was carefully constructed to determine the type and the nature of their herbal medicine use during covid 19 infection, and to find out what variables influence their decision to use herbal medicine. This survey was written in English and the questionnaire was then translated into Arabic, so that all segments of society can answer the questionnaire.

The following section of the questionnaire was divided into three parts. The first phase of the study focused on the individuals' chronic illness histories as well as socio-demographic data such age, education level, occupation, and gender. The next section is asked about the frequency, motivation, and information source of participation, frequency, pattern, duration, as well as the negative outcomes of using herbs and natural products as a form of treatment or protection during the pandemic of COVID-19.

The last part is about participant's beliefs and knowledge about herbal medicinal plants.

\section{Strategy for sampling}

This study used social media as a convenience sampling method during curfew and participants were asked to participate voluntarily. All participants received a link to the survey via Facebook and WhatsApp.

Participants in this study had to be at lived in Jordan and consumed herbs and natural products to protect themselves during the pandemic of COVID-19. Participators were not allowed to participate if neither herbs nor natural products were used as COVID19 treatment or preventative measures.

Open Epi version 3.0 was used to calculate the sample size. To achieve a $5 \%$ margin of error and a $95 \%$ confidence interval, this study requires a minimum sample size of 450 individuals.

\section{Analytical statistics}

After the info was extracted, it had been entered into the IBM SPSS version 22 statistical program (SPSS, Inc., Chicago, IL). The two-tailed tests were employed in all statistical analyses. A P-value of less than 0.05 was considered statistically significant. All variables were subjected to descriptive analysis supported frequency and percentage distributions.

All factors were examined, including demographic information, herb and natural product use rates, usage patterns, reasons to be used, and symptoms.

It was used to determine if the justifications for using herbs and natural products matched the participants' educational level. To examine the correlations, Pearson's chi-squared test or a precise probability test was used.

\section{RESULTS}

\section{Study participants' socio-demographic variables}

There were 540 participants in this study that used herbs and natural remedies.

The majority of the participants were females $458(85.5 \%)$, their age ranging from 15-25 years.

276(51.7\%) were from the capital of Jordan Amman region 192(35.7\%), and the rest came from other regions in Jordan.

Most participants 230 (42.8\%) had a bachelor's degree, while $2(0.4 \%)$ were uneducated. The vast majority of the participants $442(83.1 \%)$ did not report having any chronic conditions, while hypertension (15.7\%), diabetes (11.4\%), and cardiovascular disease (5.7\%) were reported.

During the pandemic of COVID-19, the prevalence of herbs and natural products, as well as the way of usage and frequency of use, were investigated.

The number of infected our participants and their families reached 792 , and $36.9 \%$ of them had tested PCR positive for coronavirus in the laboratories of the Ministry of Health in Jordan, and $63.1 \%$ did not take the test.

During the pandemic COVID-19, there was a statistically significant difference between people who utilized herbs and natural products $(\mathrm{P}=0.038)$.

According to the findings of the current study, 340 people said they used herbs and natural products out of a total of 540. We tested 17 types of herbs and natural products as demonstrated in figure 1.

Pimpinella Anisum (Anise 49.7\%), Matricaria chamomilla (Chamomile $45.8 \%$ ), Syzygium aromaticum (Clove 45.2\%), Mentha piperita (Peppermint 41.2\%), Zingiber officinale (Ginger 40\%), Curcuma longa (Turmeric 26.6\%), Thymus vulgaris )Thyme 20.3\%), Nigella sativa (black seeds 19.2\%), Saussurea costus (15.3\%), Psidium guajava (Guajava leaves 7.9\%), Cuminum cyminum (Cumin 7.9\%), Sesamum Indicum (Sesame 3.4\%), Brassica Alba (Mustard seeds 1.1\%), Bee propolis (14.7\%), Citrus limon (Lemon 79.7\%)were the most commonly used herbs by the participants, then Allium cepa (Onion) and Allium sativum (Garlic) respectively (59.5\%, 53.1\%), as illustrated in Figure1.

Most of our participants (45.5\%) evaluate the satisfactory response after consuming herbs and natural products were good and just $(2.1 \%)$ of participants were evaluated it with No response as demonstrated in table 2.

According to the results of the current study, (66.4\%) of participants employed herbs to enhance their immunity and (34.4\%) prevention against the COVID-19 infection, and (58.7\%) used them to relieve the symptoms and cure the ailment of the COVID-19 pandemic. 


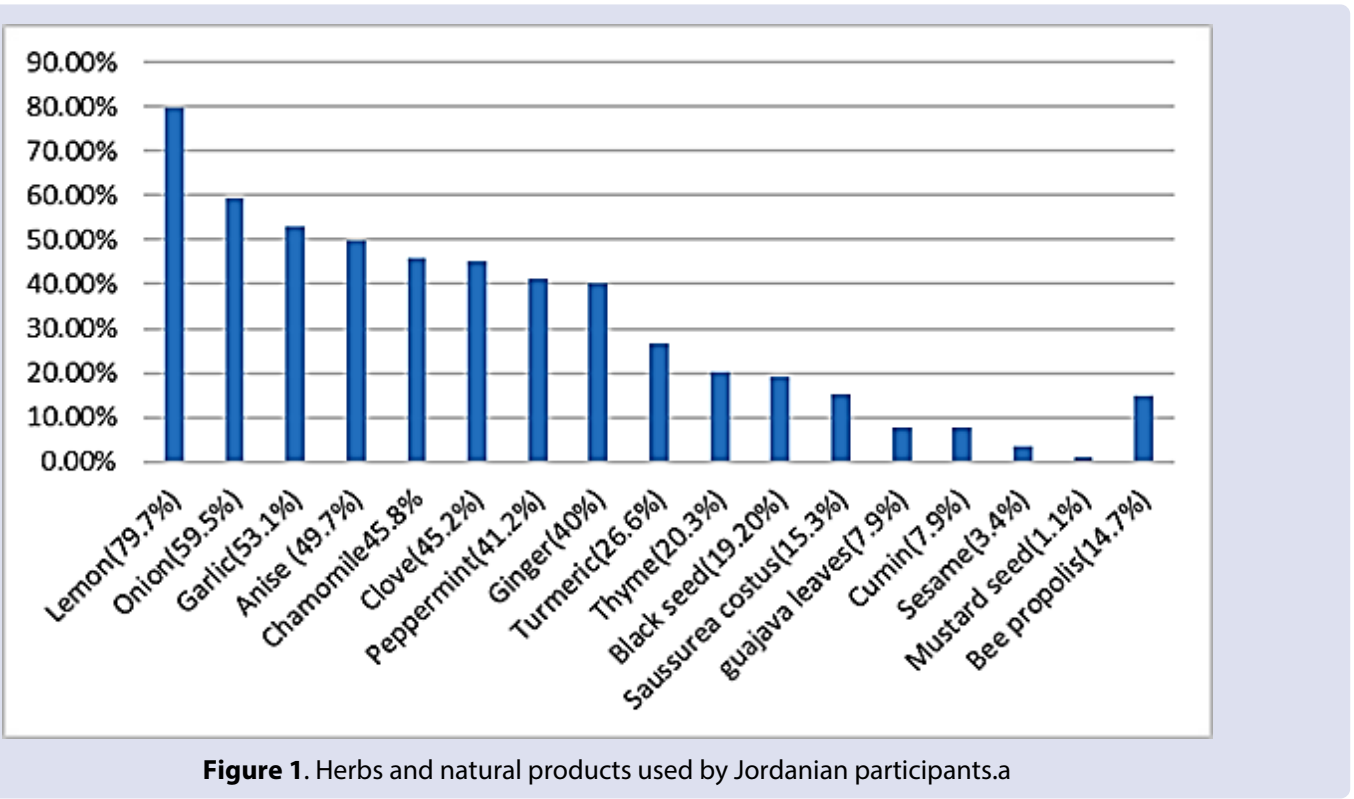

Table 1. The Sociodemographic Profile of the participants.

\begin{tabular}{lll}
\hline \multirow{3}{*}{ Gender } & & $\mathrm{N}(\%)$ \\
& male & $76(14.2)$ \\
& female & $458(85.5)$ \\
& $<14$ years & $2(0.4)$ \\
Age & $15-25$ years & $204(37.9)$ \\
& $26-35$ years & $132(24.5)$ \\
& $36-45$ years & $120(22.3)$ \\
& $46-55$ years & $62(11.5)$ \\
& $>56$ years & $18(3.3)$ \\
Educational level & Postgraduate & $122(22.7)$ \\
& undergraduate & $230(42.8)$ \\
& diploma & $141(26.4)$ \\
& High graduate school & $42(7.8)$ \\
& Uneducated & $2(0.4)$ \\
Occupation & Unemployed & $134(24.9)$ \\
& Student & $160(29.7)$ \\
Location & Employed & $234(34.5)$ \\
& Retired & $10(1.9)$ \\
& Amman & $192(35.7)$ \\
& Zarqa & $96(17.8)$ \\
& Irbid & $82(15.2)$ \\
& Aqaba & $80(14.9)$ \\
& Any other & $88(16.4)$
\end{tabular}

Table 2. Satisfactory (usefulness) outcomes after consuming Herbs and natural products during pandemic COVID-19.

\begin{tabular}{|c|c|c|}
\hline & & $\mathrm{N}(\%)$ \\
\hline \multirow{5}{*}{$\begin{array}{l}\text { Usefulness } \\
\text { (outcome) }\end{array}$} & Unsatisfied (No benefit) & $8(2.1)$ \\
\hline & Very good & $112(29.9)$ \\
\hline & Good & $170(45.5)$ \\
\hline & Moderate & $67(18.2)$ \\
\hline & Low & $15(4.3)$ \\
\hline
\end{tabular}

This suggests that the primary factor influencing the majority of the survey participants is the belief that herbs, and natural products strengthen the immune system. In light of the fact that herbs and natural products can help boost immunity and defend against COVID-19 infection.
Reasons that influenced the use of herbs by participants during the pandemic COVID-19 shown in table 3. The main three sources of herbal information were demonstrated in table $4.474(82.3 \%)$ said they used it on a regular basis, while $66(17.7 \%)$ said they used it irregularly.

According to the data analyzed on the methods and frequency of using herbs and natural products during the COVID-19 pandemic, the majority of the participants reported eating them raw (42\%), followed by raw mixed with honey (12.7\%), consuming after soaking in water for a period of time $(8.9 \%)$, and boiling in water before consumption (20.4\%). Most of the participants 216 (54.8\%) combine two or more herbs mentioned in figurel. The majority of participants utilize these herbs once per day and (17.7\%) consume it PRN especially in respiratory distress as shown in table 5 .

The difference between individuals who had adverse effects and those who did not $(\mathrm{P}=0.0285)$ was statistically significant.

Table 6 demonstrates that around 6(1.6\%) of the participants had adverse side effects from taking herbs and 20(5.3\%) were not sure of having herbal side effects, while the remaining 354(93.1\%) had no symptoms. Diarrhea was the most prevalent side effect, affecting (20\%), (11.4\%) of participants had vomiting, and abdominal pain, followed by nausea $(5.7 \%)$, drowsiness, anxiety and rash (2.9\%).

$36.3 \%$ of the respondents didn't believe that medicinal herbal treatment is better than traditional medicine in treating coronavirus 19 , while $24.9 \%$ believed in the ability of herbs to treat covid 19 more than traditional therapy.

\section{DISCUSSION}

During the pandemic COVID-19, herbs were widely used worldwide, furthermore, the majority of Arabs still use traditional herbs and natural products such as honey and propolis to maintain their health. ${ }^{18,19}$

The usage of herbs has grown in popularity in Jordan, and that is in correspond with the results of our study that there were 340 participants who used herbs and natural products out of a total of 540 .

During the COVID-19 lockdown, herbs and natural products mentioned in our study were related to educational attainment, gender, and age and that agreed with other studies. ${ }^{20}$

Information about the use of herbs from the participants' sources and natural products were inspired and recommended from family/ 
Table 3. Reasons that influenced the use and not use of herbs by participants during the pandemic COVID-19.

\begin{tabular}{lll}
\hline $\begin{array}{l}\text { Reason of use } \\
\text { Enhance their immunity }\end{array}$ & $\begin{array}{l}\mathrm{N}(\%) \\
\begin{array}{l}\text { Prevention against the COVID-19 infection, } \\
186(34.4)\end{array}\end{array}$ & $\begin{array}{l}\text { Reason for not use } \\
\text { Believe in useless of herbs versus the unexpected side } \\
\text { effects }\end{array}$ \\
$\begin{array}{l}\text { To relieve the symptoms and cure the ailment of } \\
\text { the COVID-19 pandemic. }\end{array}$ & $\begin{array}{l}\text { Not knowning enough information about these herbs } \\
\text { 3147(58.7) }\end{array}$
\end{tabular}

Table 4. Sources of herbal knowledge by participants during the COVID-19 pandemic.

\begin{tabular}{lc}
\hline Knowledge source & $\mathrm{N}(\%)$ \\
\hline family/friends & $202(54.3)$ \\
prior experience & $110(20.3)$ \\
social media & $99(18.3)$ \\
internet & $102(18.8)$ \\
Health care staff & $90(16.7)$
\end{tabular}

Table 5. The frequency and method of herbs usage throughout the COVID-19 pandemic.

\begin{tabular}{llll}
\hline Frequency & $\mathrm{N}(\%)$ & Method of use & $\mathrm{N}(\%)$ \\
\hline Once daily & $138(37.1)$ & raw & 227(42) \\
Twice daily & $104(28)$ & by raw mixed with honey & $69(12.7)$ \\
Three times daily & $36(9.7)$ & consuming after soaking in water for a period of time & $103(18.9)$ \\
More than three times daily & $28(7.5)$ & boiling in water before consumption & $380(70.4)$ \\
PRN (dyspnea) & $67(17.7)$ & inhalation & 157(29.1)
\end{tabular}

Table 6. Participants adverse effects after using herbal remedies.

\begin{tabular}{|ccc}
\hline & & N(\%) \\
\hline \multirow{2}{*}{ Side Effects } & No & $374(98.5)$ \\
& Yes & $6(1.6)$ \\
& Nausea & $4(5.7)$ \\
& Vomiting & $8(11.4)$ \\
& Abdominal pain & $8(11.4)$ \\
& Diarrhea & $14(20)$ \\
& Anxiety & $2(2.9)$ \\
& Allergy / Rash & $2(2.9)$ \\
& Drowsiness & $2(2.9)$
\end{tabular}

friends, prior experience, social media, and other sources. In contrast to Jordanian participants, people in the United States and European countries used the internet to learn about herbs and natural products during the pandemic. ${ }^{21}$ Therefore, public awareness efforts on how to find reliable information should be carried out and people's knowledge will be increased if they get information from the trustworthiness of medical information sources.

People seek herbal therapies when they have few other options and want to increase their chances of survival.

Individuals use herbs as alternative solutions to boost their immune systems and reduce their chances of developing an infection around the world.

Infections and viruses such as the common cold, influenza, fever, and even herpes have long been treated using herbal treatments. ${ }^{22}$

Our study lining up with many studies conducted in many other countries such as Thailand, China, Saudi Arabian, and Palestine respectively regarding the preference of community of using herbs and natural products instead of traditional medicinal therapy for enhancing immunity system ${ }^{23,24,25,26}$, prevent and cure of the pandemic COVID-19.

In Africa, phytomedicines are widely utilized as alternative medicines and play an important role in disease management. As a result, it's critical to assess the safety and usefulness of these indigenous botanical assets in medicine before recommending them to the medical community and the public. ${ }^{27}$

The key finding of our study is the widespread usage of herbs and more popular among people in their early twenties and thirties, particularly lemon, onion, garlic, anise, chamomile, clove, peppermint, turmeric, thyme, black seeds, ginger.

In a Korean study, four herbs from the "Ma Xing Shi Gan”, Glycyrrhizae radix, Armeniacae semen amarum, Ephedrae herbs, Gypsum fibrosum, and, were found to play a function in COVID-19. ${ }^{28}$

Significant effects of herbal medicine Had potential involvement in the treatment of COVID-19. Eucalyptus globulus. was the most mentioned plant, followed by Ziziphus lotus and Azadirachta indica. Furthermore, leaves and seeds were the most regularly employed plant parts for herbal medicines, and the majority of cures were made by infusion. ${ }^{29}$

The combined therapy increased the total effective rate, and decreased cough symptoms and sputum production symptoms. ${ }^{30,31}$

Most participants took these herbs once to twice daily and $70.4 \%$ preferred it boiled in water before consumption in combination with inhalation and the others took it raw and filled it in empty capsules.

Some herbal and natural items have been proved in previous studies to be a safe alternative treatment with few adverse effects. ${ }^{31}$ This was 
corroborated by the majority of our participants, who stated that they have used these herbs without experiencing any negative effects. However, about $1.6 \%$ of those who took part in the study had negative outcomes.

The most prevalent adverse effects reported by participants in this study were diarrhea then abdominal pain and vomiting. This contrasts with the findings of prior studies ${ }^{27,29}$ which identified a variety of side effects, with skin rash being the most common.

\section{CONCLUSIONS}

Our results showed acceptable outcomes after using herbs and natural products, in addition the beliefs related to herbal remedies have increased during pandemic COVID-19.

Most Jordanians participants preferred using herbs as potential alternatives to boost their immune system and reduce their chances of developing an infection during the lockdown.

The majority of the participants reported consuming herbs raw or mixed with honey, whether combined two or more herbs together or alone. The majority of participants utilize these herbs once per day or PRN especially in respiratory distress.

Most of the participants believed that herbs were safe, and they didn't experience any side effects.

The validity and trustworthiness of therapeutic plants should be further validated through phytochemical and pharmacological research, and false information should be monitored and controlled across various social media platforms and communities.

\section{DECLARATION OF INTERESTS}

The authors claim that they have no known financial or personal conflicts of interest that could have influenced the findings of this study.

\section{FUNDING}

There was no support for this research from any funding agency or organization.

\section{REFERENCES}

1. Weiss, Susan R., and Julian L. Leibowitz. Coronavirus Pathogenesis. Advances in Virus Research. 2011; 81: 85-164.

2. Xu, Xintian, Ping Chen, Jingfang Wang, Jiannan Feng, Hui Zhou, Xuan Li, Wu Zhong, and Pei Hao, et al. Evolution of the Novel CORONAVIRUS from the ONGOING Wuhan Outbreak and Modeling of Its Spike Protein for Risk of Human Transmission. Science China Life Sciences. 2020; 63: 457-60.

3. Qian, Jiating, Yifan Feng, and Jie Li. Comments on Cross-Species Transmission of the Newly Identified Coronavirus 2019-Ncov. Journal of Medical Virology. 2020; 92: 1437-39.

4. Poudel Adhikari, Sasmita, Sha Meng, Yuju Wu, Yuping Mao, Ruixue Ye, Qingzhi Wang, Chang Sun, et al. A Literature Review of 2019 Novel Coronavirus during the Early Outbreak Period: Epidemiology, Causes, Clinical Manifestation and Diagnosis, Prevention and Control. 2020.

5. Koh, Gavin. Faculty Opinions Recommendation of Clinical Features of Patients Infected With 2019 Novel Coronavirus IN WUHAN, CHINA. Faculty Opinions - Post-Publication Peer Review of the Biomedical Literature. 2020.

6. Otman, Haley. New Cause of Covid-19 Blood Clots Identified. University of Michigan, 2020 November 2. Available from: https:// labblog.uofmhealth.org/lab-report/new-cause-of-covid-19-bloodclots-identified.
7. Klepser, Teresa Bailey, and Michael E. Klepser. Unsafe and Potentially Safe Herbal Therapies. American Journal of HealthSystem Pharmacy. 1999; 56(2): 125-38.

8. Younis Younis, Noha Abd. The Prevalence, Attitude and Awareness of Herbal MEDICINE Products Use Among Pharmacy Practitioner in Jordan. Pharmacognosy Journal. 2019; 11(5): 1082-87.

9. Ekor, Martins. The Growing Use of Herbal Medicines: Issues Relating to Adverse Reactions and Challenges in Monitoring Safety. Frontiers in Pharmacology. 2014. Available from: https:// doi.org/10.3389/fphar.2013.00177.

10. Younis Younis, Noha Abd. Online Survey for Patient Outcomes on Hericium Erinaceous Mushroom. Pharmacognosy Journal. 2020; 12, (3): 519-25

11. James, Peter Bai, Lexina Taidy-Leigh, Abdulai Jawo Bah, Joseph Sam Kanu, Jia Bainga Kangbai, and Stephen Sevalie, et al. Prevalence and Correlates of Herbal Medicine Use among Women Seeking Care for Infertility in Freetown, Sierra Leone. EvidenceBased Complementary and Alternative Medicine. 2018: 1-11.

12. Awad, Abdelmoneim, and Dana Al-Shaye. Public Awareness, Patterns of Use and Attitudes toward Natural Health Products IN Kuwait: A Cross-Sectional Survey. BMC Complementary and Alternative Medicine. 2014; 14(1).

13. Dar-Odeh, Najla, and Osama Abu-Hammad. Herbal Remedies Use in Arab Societies. Handbook of Healthcare in the Arab World. 2020; $1-21$.

14. Wazaify, Mayyada, Izzat Alawwa, Nada Yasein, Akram Al-Saleh, and Fatma U. Afifi. Complementary and Alternative Medicine (CAM) Use AMONG Jordanian Patients with Chronic Diseases. Complementary Therapies in Clinical Practice. 2013; 19(3): 153-57.

15. Sawalha, Ansam F., Waleed M. Sweileh, Sa'ed H. Zyoud, and Samah W. Jabi. Self-Therapy Practices among University Students in PALESTINE: Focus on Herbal Remedies. Complementary Therapies in Medicine. 2008; 16(6): 343-49.

16. Dhama, Kuldeep, Khan Sharun, Ruchi Tiwari, Maryam Dadar, Yashpal Singh Malik, Karam Pal Singh, and Wanpen Chaicumpa, et al. COVID-19, an Emerging Coronavirus INFECTION: Advances and Prospects in Designing and Developing Vaccines, Immunotherapeutics, and Therapeutics. Human Vaccines \&amp: Immunotherapeutics. 2020;16(6): 1232-38.

17. Popoola, Joyce. Faculty Opinions Recommendation of REMDESIVIR and Chloroquine EFFECTIVELY Inhibit the Recently Emerged Novel Coronavirus (2019-NCoV) in Vitro. Faculty Opinions - Post-Publication Peer Review of the Biomedical Literature. 2020. Available from: https://doi.org/10.3410/f.737311964.793572693.

18. Abdullah Alotiby, Amna, and Laila Naif Al-Harbi. Prevalence of Using Herbs and Natural Products as a Protective Measure during the COVID-19 PANDEMIC among the Saudi POPULATION: An Online Cross-Sectional Survey. Saudi Pharmaceutical Journal. 2021; 29(5): 410-17.

19. Misbahud Din, Fawad Al, Abdul Waris and Fatima Zia Phytotherapeutic Options for the Treatment OF COVID-19: A Concise Viewpoint. Phytotherapy Research. 2020; 35(1): 542-42.

20. Khadka D;Dhamala MK;Li F;Aryal PC;Magar PR;Bhatta S;Thakur MS;Basnet A;Cui D;Shi S. The Use of Medicinal Plants to Prevent COVID-19 in Nepal. Journal of ethnobiology and ethnomedicine. U.S. National Library of Medicine. 2021 September 17. Avialable from: https://pubmed.ncbi.nlm.nih.gov/33832492/.

21. Günalan E, Cebiog lu _IK, Çonak Ö. The Popularity of the Dietary Supplements and Functional Foods in The Coronavirus Pandemic Among The Google Users in the USA, UK, Germany, Italy and France. Complement Ther Med. 2021 Feb 15:102682.

22. Mousa, Haider Abdul-Lateef. Prevention and Treatment of Influenza, Influenza-like Illness, and Common Cold by Herbal, Complementary, and Natural Therapies. Journal of Evidence-Based Complementary \&amp; Alternative Medicine. 2016; 22(1): 166-74. 
23. Suttinee Yuvejwattana. Thailand Clears Use of Herbal Medicine for Covid-19 Treatment. Bloomberg.com. Bloomberg. 2021 September 17

24. Evidence Aid (Multiple Reviews).org [https://evidenceaid.org/ resource/traditional-chinese-medicine-and-herbal-medicine-forcovid-19-multiple-reviews/]. Traditional Chinese Medicine and Herbal Medicine FOR Covid-19 [Added 2021 June 7].

25. Abdullah Alotiby, Amna, and Laila Naif Al-Harbi. Prevalence of Using Herbs and Natural Products as a Protective Measure during the COVID-19 PANDEMIC among the Saudi POPULATION: An Online Cross-Sectional Survey. Saudi Pharmaceutical Journal. 2021; 29(5): 410-17.

26. Pandey, Saumya. Potential of Medicinal Plants as Immunity Booster against Covid-19. Traditional Herbal Therapy for the Human Immune System. 2021; 455-74.

27. Statement on Herbal Remedies and Medicines for Prevention and Treatment of Covid-19.org [https://africacdc.org/download/ statement-on-herbal-remedies-and-medicines-for-prevention-andtreatment-of-covid-19-2/]. Africa CDC. [cited 2020 August 21].
28. Ang, Lin, Hye Won Lee, Anna Kim, Jun-Yong Choi, and Myeong Soo Lee. Network Analysis of Herbs Recommended for COVID-19: IDR. Infection and Drug Resistance. Dove Press. 2021 May 18

29. Ang, Lin, Eunhye Song, Hye Won Lee, and Myeong Soo Lee. Herbal Medicine for the Treatment of Coronavirus DISEASE 2019 (COVID-19): A Systematic Review and Meta-Analysis of Randomized Controlled Trials. Journal of Clinical Medicine. 2020; (9)5: 1583.

30. Du, Xuqin, Lipeng Shi, Wenfu Cao, Biao Zuo, and Aimin Zhou. Addon Effect of Chinese Herbal Medicine in the Treatment of Mild to Moderate Covid-19: A Systematic Review and Meta-Analysis. PLOS ONE. 2021; 16(8).

31. Khanna, Kanika, Sukhmeen Kaur Kohli, Ravdeep Kaur, Abhay Bhardwaj, Vinay Bhardwaj, Puja Ohri, Anket Sharma, Ajaz Ahmad, Renu Bhardwaj, and Parvaiz Ahmad, et al. Herbal ImmuneBoosters: SUBSTANTIAL Warriors of Pandemic Covid-19 Battle. Phytomedicine. 2021; 85:153361.

\section{GRAPHICAL ABSTRACT}

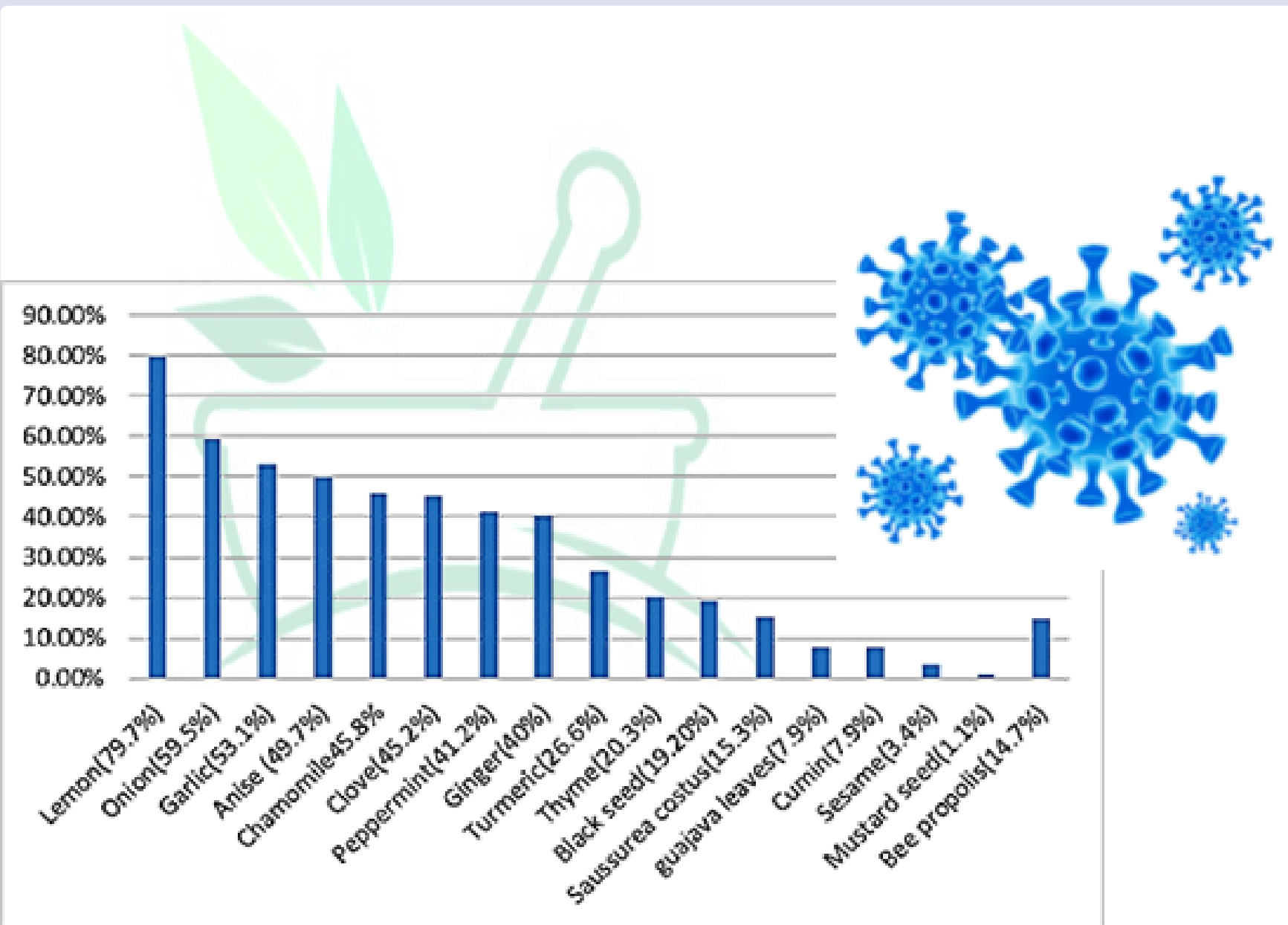




\section{ABOUT AUTHORS}

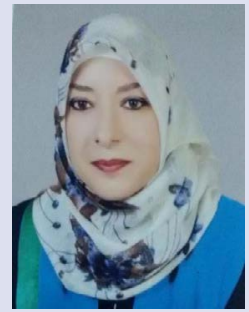

Noha Abd Alkareem Younis Younis',

1 * Department of Pharmacy, Al Balqa Applied University, Aqaba Universal College, Jordan

Email: younisnoha@bau.edu.jo

Mobile Number: 00962797773201

ORCID ID: 0000-0002-5602-7099

Rania Moh'd Hamam²,

2*Department of Pharmacy, Princess Sarvath Community College, Amman, Jordan.

Email: r.hamam@pscc.edu.jo

Mobile Number: 00962798321806

ORCID: 0000-0002-1880-5793

Amal Mayyas ${ }^{3}$,

3* Department of Pharmacy, Faculty of Health Sciences, American University of Madaba, Madaba, Jordan, P.O.Box: 2882 Amman 11821, Jordan.

Email: a.mayyas@aum.edu.jo

Mobile Number: 00962795561800

ORCID ID: 0000-0002-5036-7586

Cite this article: Younis NAAY, Hamam RM, Mayyas A. Online Survey: Prevalence and Attitude of Jordanians Towards Using Herbal Remedies in the Pandemic COVID-19. Pharmacogn J. 2021;13(6)Suppl: 1632-1638. 